\title{
Recent Advances and Future Research Directions in Edge Cloud Framework
}

\author{
Soumya Ranjan Jena, Raju Shanmugam, Rajesh Kumar Dhanaraj, Kavita Saini
}

\begin{abstract}
Recent years have shown the explosive emergence of Cloud computing in the industry and it is now the need of the hour. It is a great idea to go to utilize $5 G$ remote advancement and man-made thinking to engage speedier response times, lower latency, improved upkeep in figuring. The cloud has at no other time been so essential to the undertaking beforehand. This is where Edge Computing came into picture - seen as an expansion to the cloud, yet interesting in a couple of crucial ways. Empowering data to be taken care of, explored and moved at the edge of the framework, edge enlisting will enable undertakings to gather and assessments data closer to where it is taken care of, consistently, without idleness. Thus it can take into consideration snappy substance conveyance and information preparing that ought to be the eventual fate of registering. In this paper we will extensively study the necessity of Edge Cloud simulation environment and simulate it through EdgeCloudSim. We find that the utilization based, fuzzy competitor based and hybrid based methodologies incline toward offloading the assignments to the edge, so they give better outcomes whereas the average service time of the Fuzzy-Based methodology is least in contrast with the others.
\end{abstract} Sim

Keywords: Edge Computing, Cloud Computing, Edge Cloud

\section{INTRODUCTION}

As demonstrated by overall research and warning firm Gartner by 2025 IT organizations will convey $75 \%$ of their information outside of standard fused datacenter that is at the edge of the Cloud [1]. For over 10 years, buyer and undertaking headway clients have had their heads in the Cloud. In any case, with the dependably making uncommon principle occupations that will be added to 5G, self-rule, the Internet of Things (IoT), and interminable evaluation, affiliations are beginning to search somewhere else for their enrolling needs. We are interfacing more contraptions, and

Revised Manuscript Received on December 30, 2019.

* Correspondence Author

Soumya Ranjan Jena*, Assistant Professor, School of Computing Science and Engineering, Galgotias University, Greater Noida, Uttar Pradesh, India

Dr. Raju Shanmugam, Professor and Dean, School of Computing Science and Engineering, Galgotias University, Greater Noida, Uttar Pradesh, India.

Dr. Rajesh Kumar Dhanaraj, Associate Professor, School of Computing Science and Engineering, Galgotias University, Greater Noida, Uttar Pradesh,

Dr. Kavita Saini, Associate Professor, School of Computing Science and Engineering, Galgotias University, Greater Noida, Uttar Pradesh, India.

(C) The Authors. Published by Blue Eyes Intelligence Engineering and Sciences Publication (BEIESP). This is an open access article under the CC BY-NC-ND license (http://creativecommons.org/licenses/by-nc-nd/4.0/) crunching a bigger number of information more rapidly than at whatever point in late memory. The laziness of empowered approaches requests another method, particularly for time-central managing - in some competent city or related/driverless vehicle applications, for instance, where part second responses might be basic. Along these lines, customers and providers the proportionate are moving towards the alleged edge condition, with shippers, for instance, Dell and Microsoft setting billions of dollars in IoT portfolios and edge figuring affiliations, which move managing and information closer to the point of need. With the move of the IoT and presented understanding, the model is moving the other path for specific, applications, putting a couple of things in a colossal measure of spots, which is a totally astounding key and operational improvement challenge. Put another way, the pendulum of the IT business is swinging perpetually from bound together organizations for instance, those in the cloud - and back towards coursed systems, especially for consistent data planning in IoT, sensor group, AI, data triage, and machine-to-machine messaging applications, etc.

\section{KEY INGREDIENTS OF EDGE CLOUD ENVIRONMENT}

The key ingredients of edge computing can be outlined as follows [2]:

1. Speed: The hugest bit of leeway of edge processing is its ability to extend sort out execution by diminishing latency. Since IoT edge figuring contraptions process information locally or in close to edge server cultivates, the data they gather doesn't need to travel so far as it would under an ordinary cloud structure. By managing information closer to the source and decreasing the physical parcel it must travel, edge figuring can fabulously diminish idealness. The last thing is higher rates for end clients, with torpidity evaluated in microseconds instead of milliseconds. Taking into account that even a solitary portrayal of inertness or individual time can cost affiliations an enormous number of dollars, the speed tendencies of edge figuring can't be ignored.

2. Security: One basic worry over IoT edge computing devices is that they could be utilized as a point of portion for cyber attacks, permitting malware or different impedances to debase a structure from a solitary delicate point. While this is a legitimate hazard, the hovered idea of edge figuring arrangement makes it increasingly direct to execute security shows that can close undermined parts without closing down the whole structure.

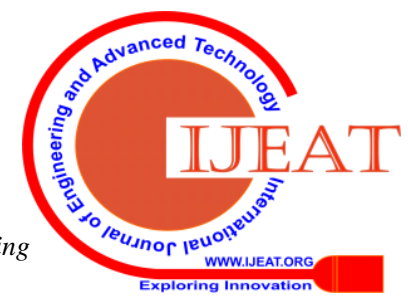


3. Scalability: Luckily, the improvement of cloud-based advancement and edge taking care of has made it less intricate than at whatever point in continuous memory for relationship to scale their endeavors. Ceaselessly, computing, storage and analytics capacities are being packaged into contraptions with tinier impressions that enable relationship to use these gadgets to build up their edge system's compass and limits.

4. Versatility: The versatility of edge figuring moreover makes it staggeringly adaptable. By banding together with close by edge data centers, associations can without a doubt target alluring markets without placing assets into exorbitant establishment augmentation. Edge data centers empower them to help end customers capably with insignificant physical partition or idleness. It is particularly important for content suppliers hoping to convey continuous gushing administrations. They likewise don't oblige organizations with an overwhelming impression, enabling them to agilely move to different markets should monetary conditions change.

5. Reliability: Given the security central focuses gave by edge dealing with, it ought not come as a frightening that it offers better determined quality in addition. With IoT edge figuring gadgets and edge server ranches organized nearer to end clients, there is less likelihood of a system issue in a distant district affecting neighborhood clients. For sure in case of a close by data center blackout, edge computing devices will keep on working adequately without anyone else in light of the fact that they handle imperative preparing capacities locally.

Apart from the above advantages edge computing has several drawbacks [1] [2].

1. Edge figuring forms and breaks down just a subset of information, disposing of crude data and fragmented bits of knowledge. Organizations must think about what level of data misfortune is adequate.

2. Since edge consists of distributed networks; security is the utmost challenge. With the expansion of the IoT, arrange associated gadgets, and implicit PCs, the open doors have expanded for assaults and malignant programmers to invade the gadgets and access delicate information.

3. Edge figuring requires progressively neighborhood equipment. For instance, IoT cameras require an inherent PC to send video information over the web just as a progressively complex registering process for increasingly advance procedure applications, for example, movement location or facial-acknowledgment calculation.

That is the reason business specialists are as of now working diligently pondering how to execute new approaches like zero trust security to guarantee that the IoT gadgets controlling edge figuring system aren't being betrayed clients and associations. With so much information being accumulated, associations can't stand to endure the danger of an information break. Luckily, a considerable lot of these security concerns can be mitigated by edge data centers that show a promise to ensuring their clients and their information. Consistence principles, for example, ISO 27001 and HIPAA/HITECH guarantee that a data center gives both can be sorted out closer to end clients. Edge structures

flexible framework and solid brand assurance.

\section{CLOUD VS. EDGE}

Clearly, sometimes in the IoT space does one size fit all? Cloud courses of action are consistently more affordable, even more prevailing, less complex to execute, organize, and scale, and move the cost weight from subsidizing to operational use. Also, there are various inclinations in taking care of data midway and off-premise with respect to transportability, remote working, facilitated exertion, and versatility. Finally, it boils down to residual weight distress and costs. What sum are customers prepared to process their extraordinary jobs that needs to be done on uncommon resources at the edge? Likewise, which segments can be managed in off hours, or dispatched off through low-hover satellites to a territory with more affordable power?

In the meantime, the continuous time of huge business joint exertion stage suppliers, for instance, Box, Salesforce.com, and others, give facilitated cloud game plans that customers find engaging and easy to use. A mix of both cloud and edge figuring suits various affiliations. Moreover, as these and different cross breed approaches increase, supporting programming will end up being always keen, choosing which outstanding burden gets dealt with where, as indicated by business technique, guidelines, financial aspects, and that's only the tip of the iceberg.

A few organizations are also using the frameworks organization building known as mist figuring, whereby as opposed to gathering and examining their information in 'the' cloud, or at the edge, it is facilitated on a neighborhood - a shelter between the responsiveness of edge processing and the centralization of distributed computing. The reasonableness focal points of this elective approach are emphatically engaging some IT boss. Be that as it may, as 5G gets typical throughout the following scarcely any years, and early use cases begin to quicken, edge processing is probably going to increase huge footing.

Outstanding tasks at hand that aren't latency specific will keep on being served from the least expensive sensible areas. Most associations would battle to coordinate the economies of scale feasible by the main cloud suppliers, for example, AWS, Google Cloud, Microsoft Azure and IBM, when facilitating information forms locally [20] [22].

\section{RESEARCH CHALLENGES}

The key research challenges are to calculate:

1. No of failed task on Edge/Cloud/Mobile.

2. No of completed tasks on Edge/Cloud/Mobile.

3. No of uncompleted task on Edge/Cloud/Mobile.

4. Percentage of failed task

5. Average service time

6. Average processing time

7. Average network delay

8. Average server utilization

This paper is further written in the following manner. Related research works are discussed in section-V. 
Section-VI gives the simulation results and analysis of EdgeCloudSim outputs. Finally, the conclusion and the future research works have been given in section-VII.

\section{RELATED WORK}

Beforehand we have chipped away at how to improve the quality-of-service parameters of Cloud [3] [4] [5] by limiting the reaction time of various burden adjusting systems that can be utilized over Cloud. Aside from that we have committed our opportunity to make sense of which power model suits well for computerized Cloud [6] utilizing Green Cloud [7] alongwith different task scheduling algorithms [8].

In [9] the author Sonmez C et.al has developed a simulator called EdgeCloudSim for execution assessment of edge processing frameworks. In [10] he has given fuzzy workload based multi-level edge figuring design with edge orchestrator. In [11] he has given the display of three potential cloudlet-helped Edge Computing models are evaluated by considering a nonexclusive generic delay-intolerant application.

In [12] Cihat Baktir et.al created Software Defined Networking (SDN) as an answer for tending to the systems administration challenges as it normally fits taking care of them [5]. The key idea of SDN is to isolate the control plane from the information plane and focus the center rationale on a product based controller. Xu Chen at al. [13] has portrayed the scattered estimation offloading basic position issue among cell phone clients as a multi-client figuring offloading game. They besides take a gander at the associate property of the game and show that the game yields a Nash balance and has the obliged improvement property.

In [14] creators have portrayed about Osmotic Computing for IoT, Edge and Cloud combination. Hyunseok Chang et. al [15] at Bell labs depicted the design of the Edge Cloud and its usage on OpenStack cloud management system. Xiaoyi Tao et.al in [16] inspected a vitality capability with execution guaranteed issue in compact edge which need lower imperativeness usage with better execution of assignments, for that we propose a vitality restricting streamlining issue for portable edge distributed computing.

Liang Tong et. al in [17] proposed a various leveled edge Cloud engineering for portable processing which empowers total of the peak loads across over various levels of Cloud servers to augment the measure of versatile outstanding burdens being served. Mahadev Satyanarayanan in [18] explained the emergence of Edge Cloud in IT sector. Authors in [19] have developed a tool for energy-aware Cloud computing known as Cloud Reports.

\section{ARCHITECTURE OF EDGECLOUDSIM}

EdgeCloudSim utilizes CloudSim to give the distributed computing capacities, for example, allotting a VM on datacenters, executing an assignment on VMs, provisioning the cloud assets, and demonstrating the power utilization of the datacenters. Notwithstanding the distributed computing abilities, EdgeCloudSim gives different unique modules to deal with the edge registering situations as portrayed in Fig. 2. EdgeCloudSim gives a deliberate building to offer assistance for a grouping of fundamental functionalities, for instance, sort out showing express to WLAN and WAN, contraption flexibility model, handy and tunable weight generator. As outlined in Fig. 2, the current EdgeCloudSim structure has five principal modules available: Core Simulation, Networking, Load Generator, Mobility and Edge Orchestrator. Each module contains a default execution that can be successfully extended. Fig.1 shows EdgeCloudSim block diagram and Fig.2 shows the relationship between EdgeCloudSim modules [9] [19].

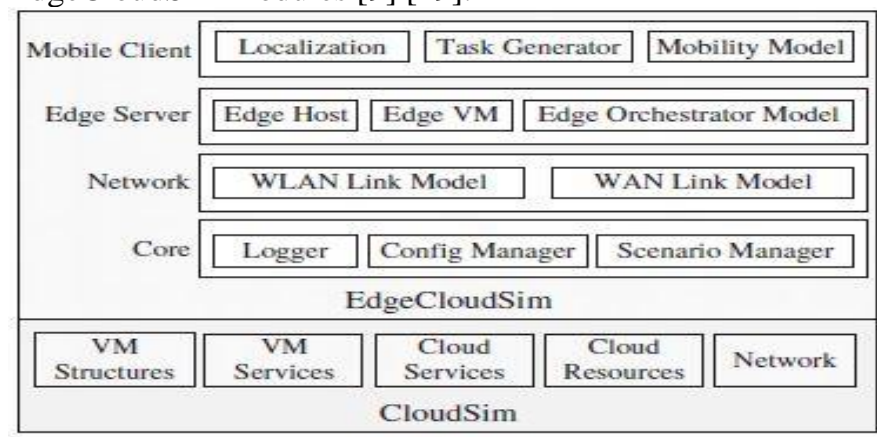

Fig.1 EdgeCloudSim Block Diagram

The core simulation module [8][18] is generally liable for stacking and running the edge enrolling circumstances from the course of action reports. EdgeCloudSim dynamically makes it possible to stack the properties of edge datacenters, the characteristics of uses, and the fundamental recreation settings. In this way, customers can run diverse propagation circumstances with unmistakable setup without changing their source code. Another huge segment of the center simulation model is the logging feature. Multiplication logs are fundamental to evaluate results. EdgeCloudSim records delayed consequences of the diversion in comma-detached worth data position. The substance of the archive and the association can be similarly changed by the prerequisites

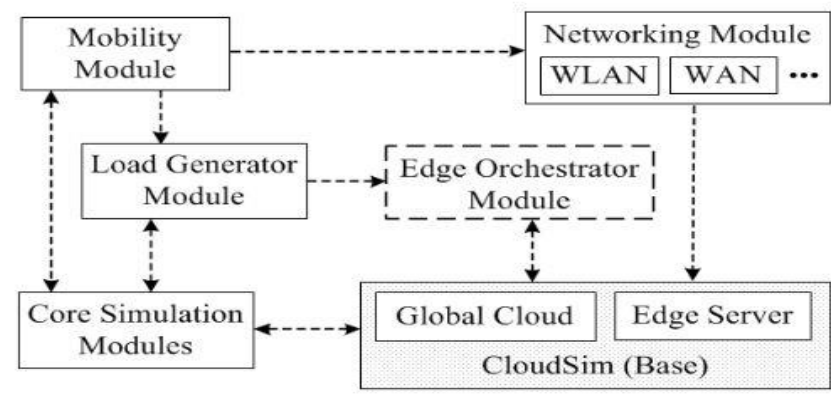

Fig.2 Edge CloudSim Modules

Fig. 3 shows the mobility module [19]. It deals with the area of edge gadgets and customers. Since CloudSim centers around the regular distributed computing

standards, the versatility isn't considered in the structure. As a matter of course, we give a traveling versatility model, however extraordinary portability models can be actualized by broadening theoretical Mobility Model class.

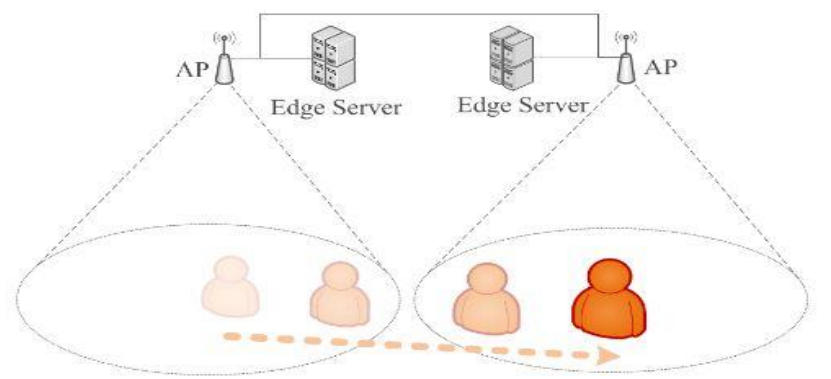

Fig.3 Mobility Module of Edge CloudSim

Blue Eyes Intelligence Engineering

\& Sciences Publication 


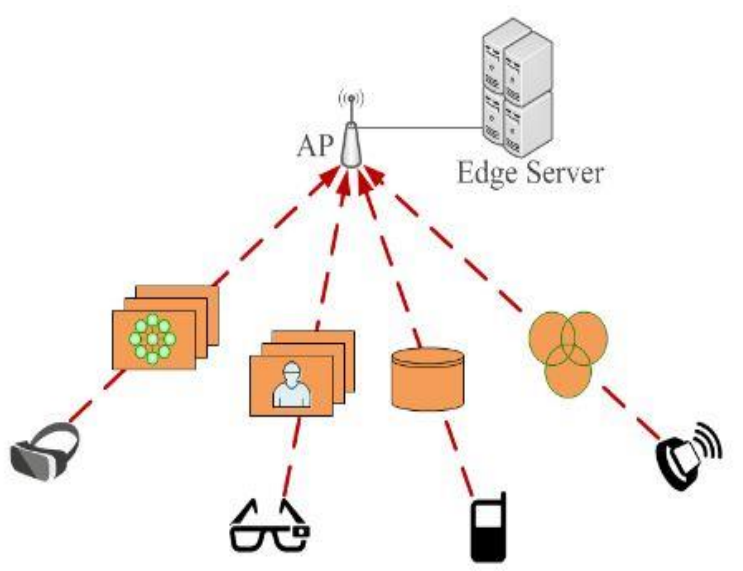

Fig.4 Load Generator Module of Edge CloudSim

The load generator module shown in Fig.4 is answerable for creating tasks for the given arrangement [19]. Of course, the tasks are produced by Poisson dissemination by means of dynamic/inert undertaking age design. In the event that other undertaking age designs are required, theoretical LoadGeneratorModel class ought to be expanded.

The Fig.5 depicts networking module.This module especially handles the transmission delay in the WLAN and WAN by considering both trade and download information [19]. The default execution of the structures association module depends on a solitary server line model.

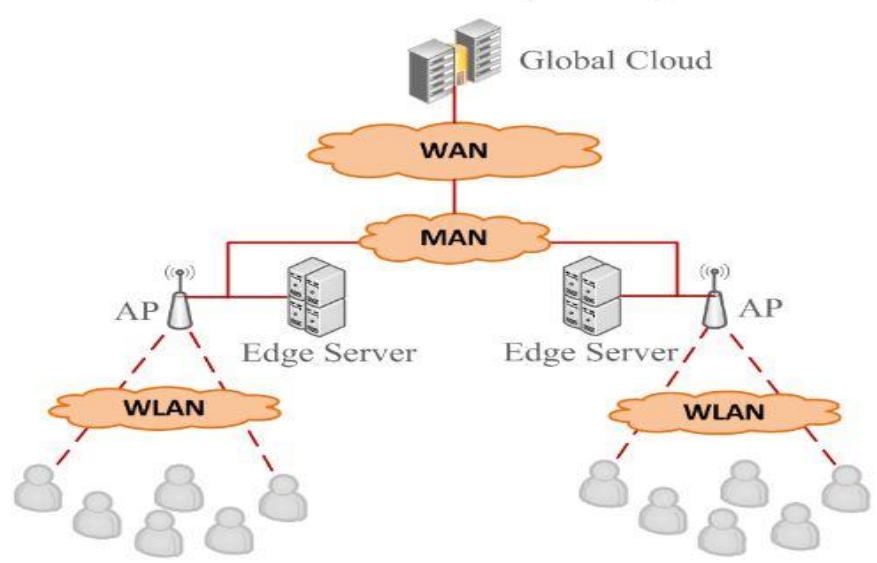

Fig.5 Networking Module of Edge CloudSim

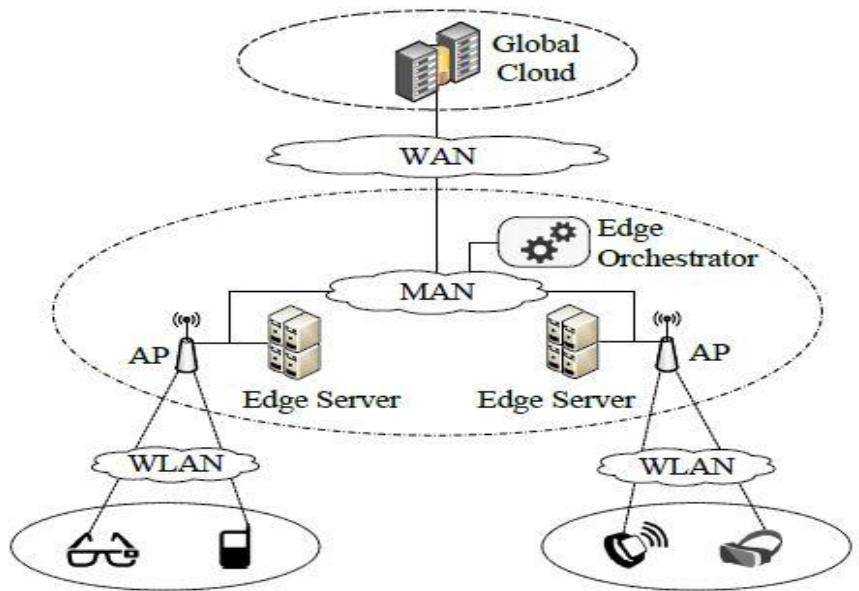

Fig. 6 Edge Orchestrator Module of Edge CloudSim The edge orchestrator module showed up in Fig.6 is the leader of the structure [9] [19]. It uses the information amassed from various modules to pick how and where to administer pushing toward client requests. In the essential change, we on a very basic level use a probabilistic strategy to manage pick where to direct pushing toward endeavors.

\section{SIMULATION ENVIRONMENT AND RESULT ANALYSIS}

We have run each examination for around 33 minutes for five occasions for 200/400/600 gadgets associated over a fundamental PC running on a Windows OS with Intel center i3-2350M CPU @2.30 GHz and 2 GB memory is utilized in the analysis. The edge figuring engineering utilized in the tests is called as two-tier with edge orchestrator that comprises of the mobile devices, edge servers, and cloud servers. They are given as follows:

1. Two-tier with Edge Orchestrator Policy Network based

2. Two-tier with Edge Orchestrator Policy Utilization based

3. Two-tier with Edge Orchestrator Policy Fuzzy based

4. Two-tier with Edge Orchestrator Policy Fuzzy Competitor based

5. Two-tier with Edge Orchestrator Policy Hybrid based Here we have just viewed as two-tier architecture over single level design since a portion of the assignments can be offloaded to the cloud servers. Generally, two-level design is marginally superior to the single level since $10 \%$ of the tasks are sent to the cloud. In addition, two-level designs give better WLAN delay execution [9] compared to the single-tier design. The LAN delay in the two-level with edge orchestrator design is becoming quicker than the two-level architecture. By using EdgeCloudSim we perform here 15 numbers of experiments consisting of 200 devices connected, 400 devices connected and 600 devices connected. During simulation each time we compute the following things:

1. No of Tasks (NT)

2. No of Failed Tasks (NFT)

3. No of Completed Tasks (NCT)

4. No of Uncompleted Tasks (NUT)

5. No of Failed Tasks due to Network (NFTN)

6. Percentage of Failed Tasks (PFT)

7. Average Service Time (AST)

8. Average Processing Time (APT)

9. Average Network Delay (AND)

10. Average Server Utilization (ASU)

The following tables 1, 2 and 3 tell us about the first five parameters i.e. NT, NFT, NCT, NUT and NFTN.

Table 1: Experiments for no of Connected Devices are 200 


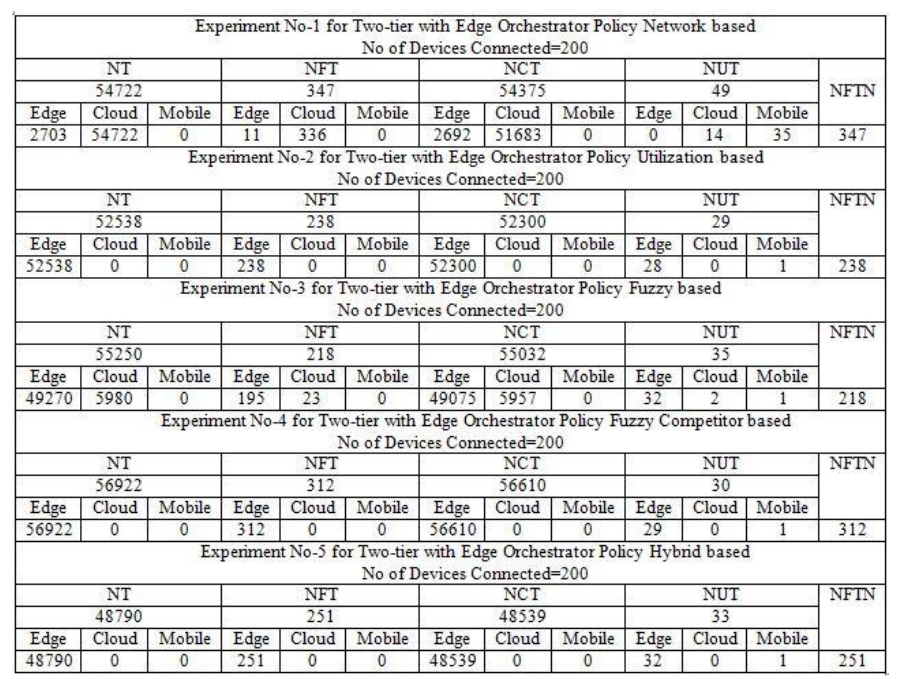

Table 2: Experiments for no of Connected Devices are 400

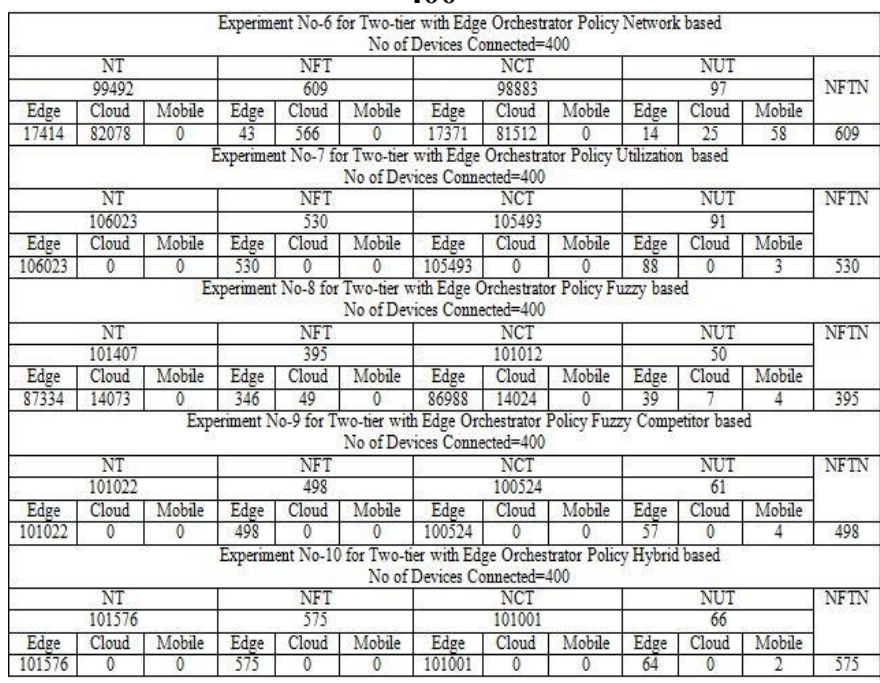

Table 3: Experiments for no of Connected Devices are 600

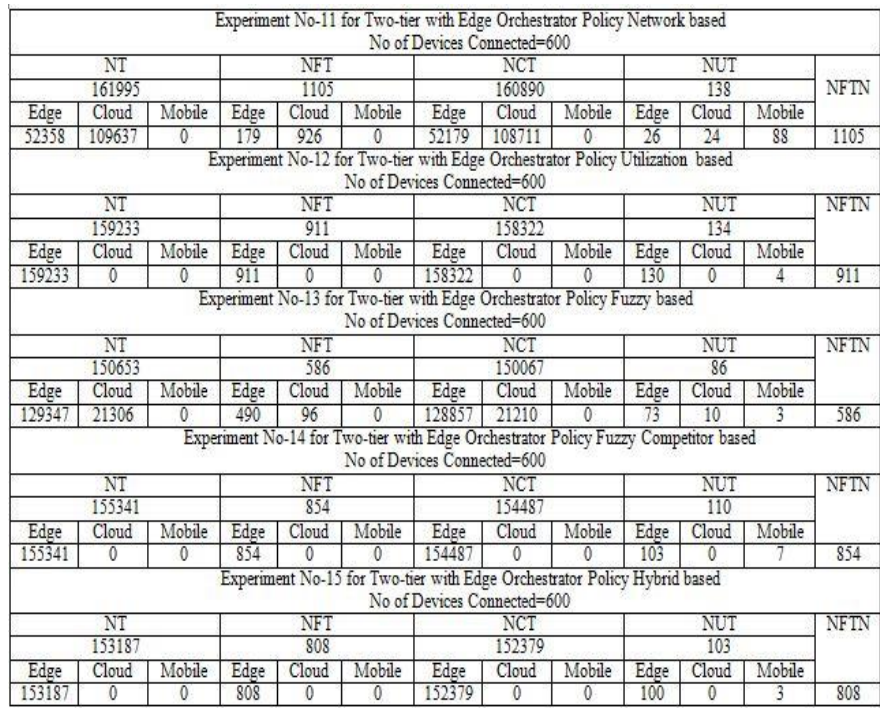

Fig. 7 tells us about \% of failed tasks (PFT), average service time (AST), average processing time (APT), and average network delay (AND) in each experiment.

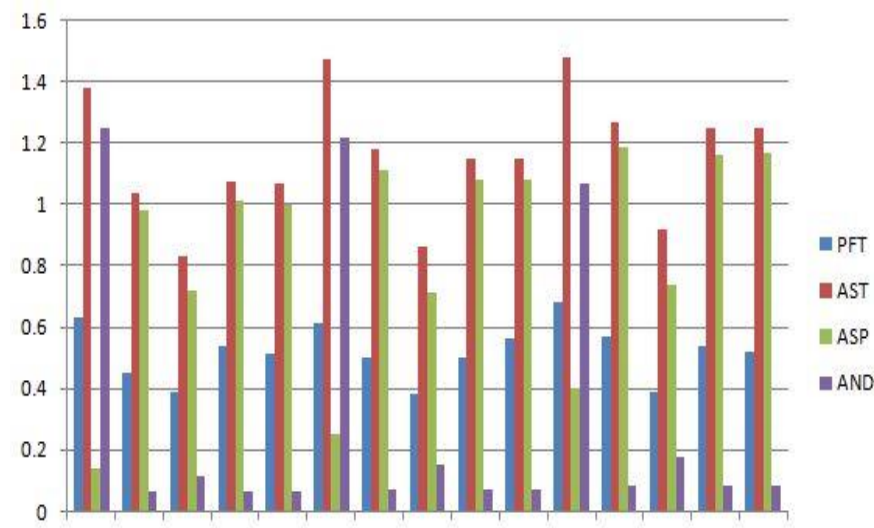

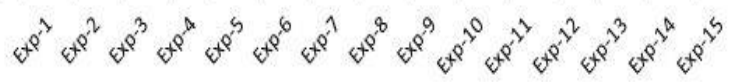

Fig. 7 Values of PFT, AST, APT and AND

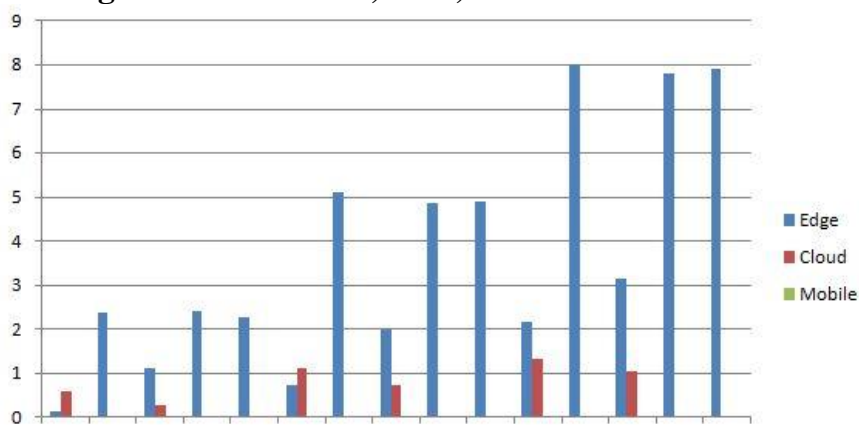

Fig. 8 Average Server Utilization in each Case The utilization based, fuzzy competitor based and hybrid based methodologies incline toward offloading the assignments to the edge, so they give better outcomes. The Network-Based and fuzzy based calculations choose to offload to the cloud because of the blockage on CPU assets on the edge servers. Since the WAN delay is higher than the processing time, they give the most exceedingly terrible presentation. It tends to be contended that the average service time of the Fuzzy-Based methodology is least in contrast with the others.

\section{CONCLUSION AND FUTURE WORK}

Moving data taking care of to the edge of the framework can help associations with abusing the creating number of IoT edge contraptions, improve organize speeds, and update customer encounters. The flexible thought of edge handling furthermore makes it an ideal response for rapidly creating, deft associations, especially in the occasion that they're starting at now using collocation data centers and cloud establishment. By abusing edge handling, associations can improve their frameworks to give versatile and trustworthy help that will bolster their picture and keep their customers energetic.Edge figuring offers a few points of interest over customary types of system engineering and will most likely keep on assuming a significant job for organizations going ahead.

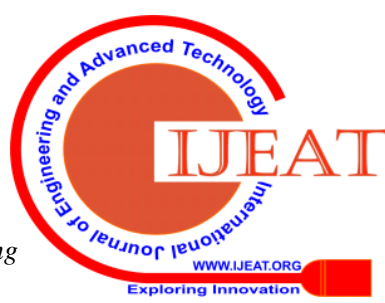


With increasingly more web associated gadgets hitting the market, imaginative associations have likely just start to expose what's conceivable with edge figuring.

\section{REFERENCES}

1. https://indianexpress.com/article/explained/from-the-cloud-computingmoves-to-the-edge-6093733/. Accessed Nov, 14. 2019

2. https://www.vxchnge.com/blog/the-5-best-benefits-of-edge-computing. Accessed Nov, 14. 2019.

3. Soumya Ranjan Jena, and Zulfikhar Ahmad, "Response Time Minimization of Different Load Balancing Algorithms in Cloud Computing Environment", IJCA, Vol 69, No. 17, Pages 22-27, May 2013.

4. Soumya Ranjan Jena, and Bhushan Dewan, "Improving Quality-of-Service Constraints of Cloud Data Centers", IEEE, 2nd International Conference on Computing for Sustainable Global Development, BVICM, New Delhi ,2015.

5. Soumya Ranjan Jena, Sudarshan Padhy, and Balendra Kumar Garg, "Performance Evaluation of Load Balancing Algorithms on Cloud Data Centers", IJSER, Vol 5, 3, Pages 1137-1145, 2014.

6. Soumya Ranjan Jena, V. Vijayaraja, and Aditya Kumar Sahoo, "Performance Evaluation of Energy Efficient Power Models for Digital Cloud”, INDJST, Vol 9, 48, Pages 1-7, 2016.

7. Soumya Ranjan Jena, and L.Shridhara Rao,“ A Study on Energy Efficient Task Scheduler over Three-Tier Cloud Architecture using Green Cloud", JARDCS, Vol 9, 18, 2017.

8. Soumya Ranjan Jena, Sukant Kishoro Bisoy and Bhushan Dewan, "Performance Evaluation of Energy Efficient Power Models for Differnent Scheduling Algorithms in Cloud using Cloud Reports", IEEE, GUCON 2019, Galgotias University, Greater Noida, U.P, India.

9. Sonmez C, Ozgovde A, Ersoy C., "EdgeCloudSim: An Environment of Performance Evaluation of Edge Computing Sysems", Trans Emerging Tel Tech. 2018;29:e3493, https://doi.org/10.1002/ett.3493.

10.Sonmez C, Ozgovde A, Ersoy C., "Fuzzy Workload Orchestration for Edge Computing", IEEE Transactions on Network and Service Management, DOI 10.1109/TNSM.2019.2901346.

11.Sonmez C, Ozgovde A, Ersoy C., "Performance Evaluation of Single-Tier and Two-Tier Cloudlet Assisted Applications" ICC2017: WS07-Workshop on Flexible Networks (FlexNets).

12.Cihat Baktir, Cagatay Sonmez, Cem Ersoy, Atay Ozgovde, and Blesson Varghese, "Chapter 2- Addressing the Challenges in Federating Edge Resources", Wiley STM (Editor Buyya, Srirama), Fog and Edge Computing: Principles and Paradigms.

13.Xu Chen, Member, Lei Jiao, Wenzhong Li, and Xiaoming Fu, "Efficient Multi-User Computation Offloading for Mobile-Edge Cloud Computing", IEEE/ACM Transactions on Networking ( Volume: 24 , Issue: 5 , October 2016 .

14.Massimo Villari and Maria Fazio, Schahram Dustdar, Omer Rana and Rajiv Ranjan, "Osmotic Computing: A New Paradigm for Edge/Cloud Integration”, IEEE COMPUTER SOCIETY, 2016.

15.Hyunseok Chang Adiseshu Hari Sarit Mukherjee T.V. Lakshman, "Bringing the Cloud to the Edge", Bell Labs, USA.

16.Xiaoyi Tao, Kaoru Ota, Mianxiong Dong, Heng Qi, and Keqiu Li, "Performance Guaranteed Computation Offloading for Mobile-Edge Cloud Computing", IEEE Wireless Communications Letters ( Volume: 6, Issue: 6 , Dec. 2017.

17.Liang Tong, Yong $\mathrm{Li}$ and Wei Gao, "A Hierarchical Edge Cloud Architecture for Mobile Computing", IEEE INFOCOM 2016 - The 35th Annual IEEE International Conference on Computer Communications.

18.Mahadev Satyanarayanan, "The Emerengence of Egde Computing", IEEE Computer Socity, IEEE, 2017.

19.EdgeCloudSim: https://github.com/CagataySonmez/EdgeCloudSim. Accessed Nov, 14. 2019.

20.Rajkumar Buyya, Rajiv Ranjan, Rodrigo N. Calheiros, "Modeling and Simulation of Scalable Cloud Computing Environments and the CloudSim Toolkit: Challenges and Opportunities", 2009 International Conference on High Performance Computing \& Simulation, Leipzig, Germany, 21-24 June 2009.

21.Thiago Teixeira Sá, Rodrigo N. Calheiros and Danielo G. Gomes, "Chapter 6: CloudReports: An Extensible Simulation Tool for Energy-Aware Cloud Computing Environments", Springer International Publishing Switzerland, Cloud Computing, Computer Communications and Networks, page 127-141, 2014..

22.R.Buyya, C. S. Yeo, S. Venugopal, J. Broberg, and I. Brandic, "Cloud Computing and Emerging IT Platforms: Vision, Hype, and Reality for Delivering Computing as the 5th Utility. Future Generation Computer
Systems", 25(6): 599-616, Elsevier Science, Amsterdam, The Netherlands, June 2009.

23.R. Buyya and M. Murshed, "GridSim: A Toolkit for the Modeling and Simulation of Distributed Resource Management and Scheduling for Grid Computing", Concurrency and Computation: Practice and Experience, 14(13-15), Wiley Press, Nov.-Dec., 2002.

24.Rodrigo N. Calheiros, Rajiv Ranjan, Anton Beloglazov, C'esar A. F. De Rose, and Rajkumar Buyya, "CloudSim: A Toolkit for Modeling and Simulation of Cloud Computing Environments and Evaluation of Resource Provisioning Algorithms", John Wiley \& Sons, SoftwarePractice and Experience, 41, Pages 23-50, 2011.

25.Bhathiya Wickremasinghe, "CloudAnalyst: A CloudSim based Tool for Modelling and Analysis of Large Scale Cloud Computing Environments" MEDC project report, 433-659 Distributed Computing project, CSSE department., University of Melbourne, 2009.

26.Thaigo Teixeira Sa, Rodrigo N Calheiros and Danielo G Gomes, "CloudReports: An Extensible Simulation Tool for Energy-Aware Cloud Computing Environments", Springer International Publishing. Chapter 6, Cloud Computing- Challenges, Limitations and R \& D Solutions. $201421^{\text {st }}$ Oct (Book); p. 127-42,. ISBN: 978-3-319-10529-1.

27.Minxian Xu, Wenhong Tian, Xinyang Wang, and Qin Xiong, "FlexCloud: A Flexible and Extendable Simulator for Performance Evaluation of Virtual Machine Allocation", 2015 IEEE International Conference on Smart City/SocialCom/SustainCom (SmartCity), 19-21 Dec. 2015, Chengdu, China.

28.Beloglazov A. Department of Computing and Information Systems, The University of Melbourne: "Energy-efficient Management of Virtual Machines in Data Centers for Cloud Computing", Doctor of Philosophy (PhD Thesis), 2013 February.

29.Wenhong Tian, Yong Zhao, Minxian Xu, Yuanliang Zhong, Xiashuang Sun, "A Toolkit For Modeling and Simulation of Real-time Virtual Machine Allocation in a Cloud Data Center", IEEE Transactions on Automation Science and Engineering, Volume: 12 , Issue: 1, Jan. 2015.

30.Núñez, Alberto, Vázquez-Poletti, Jose Caminero, Agustín Castañé, Gabriel Carretero, Jesus Llorente, Ignacio, "ICanCloud: A Flexible and Scalable Cloud Infrastructure Simulator", Journal of Grid Computing. 10. 185-209. 10.1007/s10723-012-9208-5, 2012. 